\title{
Glutathione S-transferases in neonatal liver disease
}

\author{
J Mathew, A R Cattan, A G Hall, J E Hines, R Nelson, E Eastham, A D Burt
}

\begin{abstract}
Aims: To investigate the distribution of a and $\pi$ class glutathione S-transferases (GST) in normal fetal, neonatal, and adult liver; and to examine changes in GST expression in neonatal liver disease. Methods: $a$ and $\pi$ class GST were immunolocalised in sections of formalin fixed liver tissue obtained from human fetuses $(n=21)$, neonates $(n=8)$, young children $(n=9)$ and adults $(n=10)$, and from neonates with extrahepatic biliary atresia $(n=15)$ and neonatal hepatitis $(n=12)$. Monospecific rabbit polyclonal antibodies were used with a peroxidaseantiperoxidase method.

Results: Expression of $\pi$ GST was localised predominantly within biliary epithelial cells of developing and mature bile ducts of all sizes from 16 weeks' gestation until term and in neonatal and adult liver. Coexpression of $\pi$ and $a$ GST was seen in hepatocytes of developing fetal liver between 16 and 34 weeks' gestation. Although $\pi$ GST was seen in occasional hepatocytes up to six months of life, this isoenzyme was not expressed by hepatocytes in adult liver. By contrast, $a$ GST continued to be expressed by hepatocytes in adult liver; this isoenzyme was also seen in some epithelial cells of large bile ducts in adult liver. No change was observed in the distribution of $a$ GST in either neonatal hepatitis or extrahepatic biliary atresia. However, aberrant expression of $\pi$ GST was identified in hepatocytes of all but one case of extrahepatic biliary atresia but in only two cases of neonatal hepatitis.

Conclusions: The phenotypic alterations noted in extrahepatic biliary atresia may result from the effect of cholate stasis. Evaluation of the pattern of $\pi$ and $a$ GST distribution by immunohistochemical staining may provide valuable information in distinguishing between these two forms of neonatal liver disease.
\end{abstract}

Glutathione S-transferases (GST) are a family of isoenzymes that have multifunctional biological roles in the detoxification of xenobiotics and lipophilic ligands; they have overlapping substrate specificities and function as cytosolic binding proteins. ${ }^{1-4}$ The cytosolic GST are homo- and heterodimeric, chemically inducible isoenzymes ${ }^{34}$ which are the products of three distinct autosomal loci, GST1, GST2, and GST $3,{ }^{56}$ and are classified as near neutral $(\mu)$, basic $(a)$, and acidic $(\pi)$ on the basis of their electrophoretic mobility. ${ }^{4}$

GST are present in greatest concentration in the liver where they comprise up to $10 \%$ of cytosolic protein. $^{3}$ Serum and plasma concentrations of GST change in a variety of liver diseases, and have been shown to be a more sensitive index of acute hepatocellular damage than conventionally used biochemical markers, including aminotransferases and $\gamma$ glutamyl transferase. ${ }^{7-12}$ These include alcoholic cirrhosis, ${ }^{10}$ autoimmune hepatitis, ${ }^{11}$ and paracetamol overdose. ${ }^{12}$ Until recently, identification and measurement of GST subtypes have involved the use of starch gel electrophoresis and chromatofocusing. ${ }^{4}$ The development of antibodies to GST ${ }^{13}$ has enabled investigators to study the distribution of the different isoenzymes in normal and diseased adult human liver. Immunohistochemical studies have shown that the distribution of GST may be changed in alcoholic cirrhosis ${ }^{16}$ and malignant liver tumours. ${ }^{17}$

In this study we examined the immunohistochemical pattern of GST isoenzyme expression in the developing fetal and neonatal liver and compared this with that of the adult. We also investigated changes in the expression of these isoenzymes in two common neonatal liver diseases, neonatal hepatitis and extrahepatic biliary atresia. $^{18-21}$

\section{Methods}

Formalin fixed liver tissue from fetuses ( $n=21 ; 16$ weeks' gestation to term), neonates $(n=8)$, children $(n=9 ; 3$ months to 5 years of age) and adults $(n=10)$ were used as normal controls to examine GST expression in the developing liver. Biopsy specimens were also obtained from 27 jaundiced babies aged 6 to 8 weeks in which diagnosis of extrahepatic biliary atresia $(n=15)$ and idiopathic neonatal hepatitis $(n=12)$ was subsequently made on clinical and histopathological grounds; in the latter group no underlying infective or metabolic disorders could be detected in any case. Biopsy specimens were stained with conventional histochemical stains: haematoxylin and eosin, picromallory, reticulin, Shikata's orcein, and diastase periodic acid Schiff and $3 \mu \mathrm{m}$ sections were obtained for immunohistochemical studies.

Two primary antibodies were used in this study: (i) rabbit polyclonal anti- $\pi$ GST, dilution 1 in 400 in $20 \%$ normal swine serum 
(NSS) in TRIS-buffered saline (TBS); and (ii) rabbit polyclonal anti- $a$ GST, dilution 1 in 500 in NSS/TBS. Full details of the generation and specificity of these antibodies are given elsewhere. ${ }^{1314}$ In brief, $\pi$ GST was derived from the splenic tissue of a patient with chronic lymphocytic leukaemia. a GST was isolated from human liver. These isoenzymes were purified using affinity chromatography and fast protein liquid chromatography using a Mono $Q$ column (Pharmacia). Their substrate specifiicities, isoelectric points, and molecular weights were shown to be similar to those
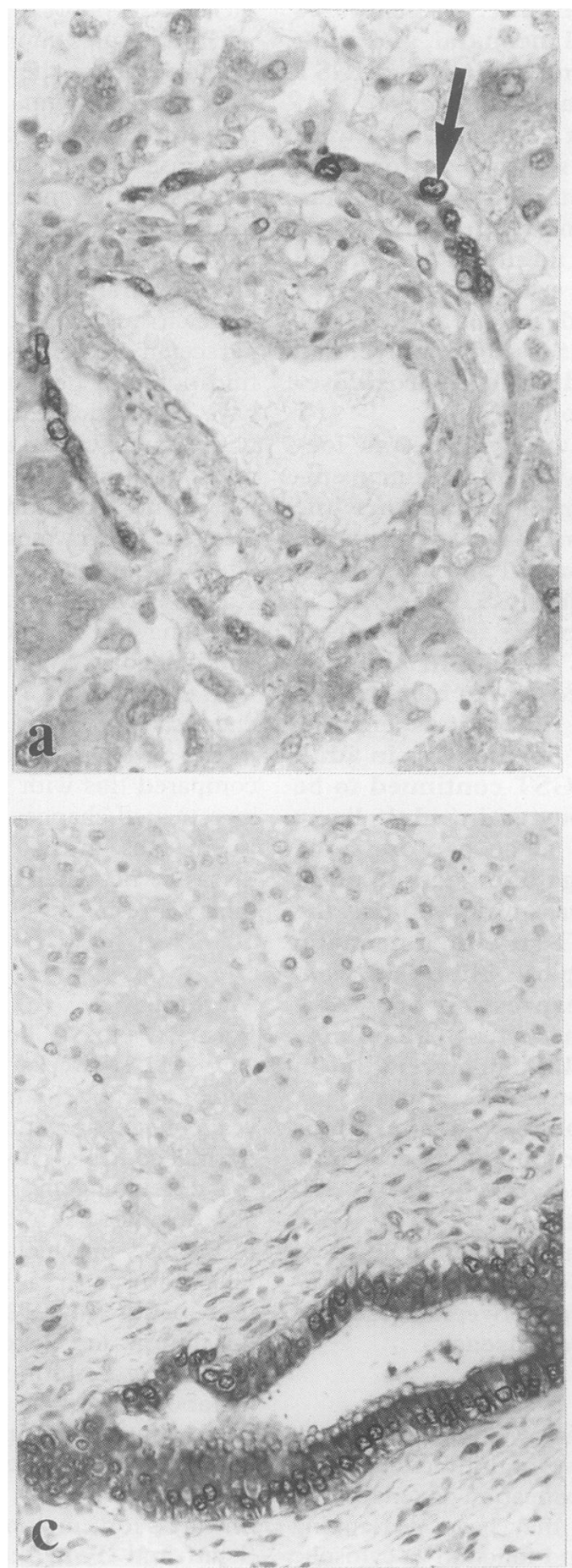

described for human placental $\pi$ and hepatic $\alpha$ forms. Polyclonal antiserum to $\alpha$ and $\pi$ GST was produced by a standard immunisation protocol in male New Zealand white rabbits. Protein $(150-200 \mu \mathrm{g})$ was introduced by intramuscular injection into the hind quarter on each occasion using Freund's complete adjuvant, followed by monthly injections using Freund's incomplete adjuvant. Immunoblotting and ELISA were used to assess antibody titre and specificity. Antisera to $a$ and $\pi$ GST isoenzymes did not cross-react with other forms of GST or other cytosolic proteins at

Figure 1 Distribution of glutathione S-transferases in $(A, B)$ fetal (18-20 weeks' gestation) and $(C-D)$ adult liver: (A) $\pi$ GST immunoreactivity can be seen in hepatocytes with intense staining of cells within the ductal plate (arrow). (B) a-GST immunoreactivity is present in hepatocytes throughout the acinus with striking nuclear labelling. Bile ducts (B) are negative. (C) $\pi$ GST immunoreactivity is not seen in adult hepatocytes but striking staining is noted in bile duct epithelium. (D) Intense cytoplasmic and nuclear stainiong for a GST is seen in adult liver. 

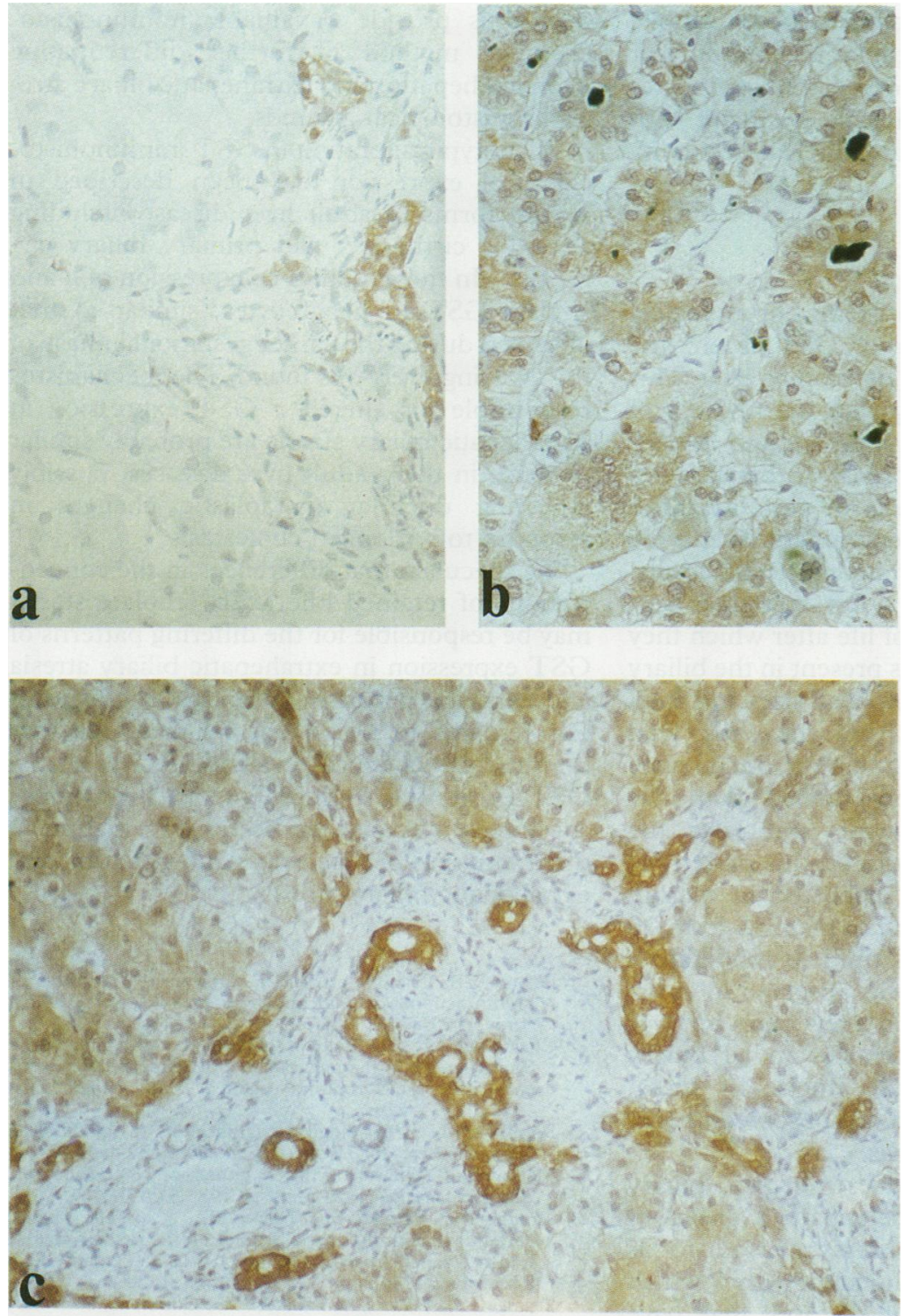

Figure 2 Glutathione S-transferases in neonatal liver disease: (A) $\pi G S T$ immunoreactivity in neonatal hepatitis: a similar pattern to that seen in normal neonatal and adult liver is present. (B) In extrahepatic biliary atresia, hepatocytes, including multinucleate cells, show expression of $\pi$ GST. (C) $\pi$ GST immunoreactivity is seen here in hepatocytes, proliferating ductules, and bile ducts in a case of extrahepatic biliary atresia. counterstained with haematoxylin, and mounted in DPX.

\section{Results}

GST EXPRESSION IN FETAL, NEONATAL, AND ADULT LIVER

$\pi G S T$

At sixteen weeks' gestation almost all heptocytes in all zones of the acinus showed cytoplasmic and nuclear positivity (fig 1A). The intensity of immunoreactivity in hepatocytes decreased towards term. After 34 weeks only occasional hepatocytes expressed this isoenzyme. In infants after 6 months and in adult liver there was no expression of this isoenzyme by hepatocytes (fig 1C). The ductal plate structures at the porto-parenchymal interface in the second trimester (from 16 weeks' gestation) (fig 1A), as well as the developing and mature bile ducts of all sizes after this period and in adult liver showed intense nuclear and cytoplasmic immunoreactivity (fig 1C). Intra-sinusoidal haematopoietic cells did not contain this isoenzyme.

\section{a GST}

Hepatocytic nuclear and cytoplasmic $a$ GST immunoreactivity was seen at 16 weeks' gestation throughout the acinus (fig 1B). This pattern of staining was present throughout fetal development and in adult liver (fig 1D). Between 16 and 24 weeks' gestation there was mild nuclear and cytoplasmic positivity in some cells in intermediate sized bile ducts, although most bile ducts and ductal plate structures were negative. Later, only very occasional cells in larger bile ducts showed intense cytoplasmic, nuclear, and membrane positivity to term and in adults. The primitive intrasinusoidal haematopoietic cells were negative.

GST ISOENZYME EXPRESSION IN NEONATAL LIVER DISEASE

Neonatal hepatitis

In all 12 cases of neonatal hepatitis immunolocalisation of $a$ GST was identical with that seen in normal neonatal and adult liver. In 10 cases immunoreactivity for $\pi$ GST did not show any variation from that expected in mature livers: bile ducts of all sizes were immunoreactive and hepatocytes were negative (fig 2A). However, in two cases some hepatocytes including giant cells showed moderate cytoplasmic positivity. Histologically, no apparent differences in the degree of bilirubinostasis could be seen between these cases and the remaining 10 .

\section{Extraheptic biliary atresia}

$a$ GST isoenzyme did not show any variation from that expressed in mature adult livers. There was intense nuclear and cytoplasmic positivity in hepatocytes, although there was an apparent decrease in the number of nuclei that were positive. Proliferating ductules were negative, even in those cases of extrahepatic biliary atresia with severe bridging fibrosis.

$\pi$ GST showed a striking difference from the mark), diluted 1 in 50 in NSS/TBS for 30 minutes at room temperature; (vii) washed in TBS and incubated with 3,3'-diaminobenzidine (DAB) in the presence of hydrogen peroxide; and (viii) washed in tap water, 
expected pattern of immunoreactivity. All bile ducts showed intense cytoplasmic and nuclear positivity and, in all but one case, hepatocytes showed moderate cytoplasmic positivity with variable mild to moderate nuclear positivity (figs $2 \mathrm{~B}$ and $\mathrm{C}$ ).

\section{Discussion}

We have shown a consistent chronological variation in the pattern of GST isoenzyme expression in the normal developing liver. This observation is in keeping with the findings of Hiley $e t a l^{22}$ and Hayes et $a l^{23} a$ and $\pi$ GST immunoreactivity was intense in hepatocytes between 16 and 24 weeks of gestation after which $a$ GST was the predominant isoenzyme expressed in these cells in neonates and adults. $\pi$ GST was positive in a few scattered hepatocytes until 6 months of life after which they were negative. $\pi$ GST was present in the biliary epithelial cells of bile ducts of all sizes and at all stages of development from the early fetal period onwards. In early gestation, ductal plate structures were intensely stained with the antibody to this isoenzyme and later the developing bile ducts acquired this pattern of immunoreactivity which persisted in mature adult bile ducts of all sizes. In material from 16 and 20 week old fetuses mild to moderate $a$ GST immunoreactivity was observed in intermediate sized bile ducts in a few cases but was consistently negative after this in all biliary radicles except large bile ducts. In these, intense $a$ GST expression was observed in only some biliary epithelial cells.

Neonatal hepatitis and extrahepatic biliary atresia are thought to belong to a group of infantile bile duct disorders referred to as "idiopathic obstructive cholangiopathy" which represent a spectrum of diseases that have overlapping clinico-histomorphological features. ${ }^{180}$ Although current concepts suggest that neonatal hepatitis and extrahepatic biliary atresia may therefore overlap, their management and prognosis are different and dependent on early accurate histological diagnosis. ${ }^{181924}$ Early surgical intervention, before 60 days of life, in the form of portoenterostomy shows an improvement in the morbidity and mortality that is associated with untreated or undiagnosed extrahepatic biliary atresia, ${ }^{24}$ whereas operative intervention in neonatal hepatitis is contraindicated. Although distinction between extrahepatic biliary atresia and neonatal hepatitis can usually be made on the basis of changes in portal tract areas, this may be difficult in biopsy specimens obtained at early time points.

We have found a remarkable difference in GST immunohistochemical expression from the normal pattern in cases of extrahepatic biliary atresia. In $93 \%$ of cases there was almost universal coexpression of $a$ and $\pi$ GSTs within hepatocytes; only one case of extrahepatic biliary atresia failed to show $\pi$ GST expression. In contrast, only two cases of neonatal hepatitis $(17 \%)$ showed coexpression of these isoenzymes in hepatocytes. This striking difference in GST isoenzyme expression may thus provide a valuable immunohistochemical method of further differentiating neonatal hepatitis and extrahepatic biliary atresia on histological grounds.

Phenotypic alteration in GST immunohistochemical expression has been described in several forms of adult liver disease including alcoholic cirrhosis ${ }^{16}$ and primary biliary cirrhosis. ${ }^{17}$ In these studies coexpression of $a$ and $\pi$ class GST in hepatocytes, similar to that observed during the earlier gestational phase of the evolving liver, was found. The mechanisms responsible for altered $\pi$ GST expression in extrahepatic biliary atresia are probably similar to those in these adult liver diseases, possibly reflecting ductular metaplastic changes in response to prolonged cholestasis.

We speculate that differences in the concentrations of retained bile acids (cholate stasis) may be responsible for the differing patterns of GST expression in extrahepatic biliary atresia and neonatal hepatitis. Although this hypothesis remains to be tested, we conclude that immunohistochemical evaluation of $a$ and $\pi$ GST expression in percutaneous liver biopsy specimens may provide useful information in differentiating between neonatal hepatitis and extrahepatic biliary atresia.

1 Jakoby WB. The glutathione S-transferases: a group of multifunctional detoxification proteins. Adv Enzymol Re Areas Mol Biol 1977;47:383-414.

2 Chasseaud LF. The role of glutathione and glutathione S-transferases in the metabolism of chemical carcinogens and other electrophilic agents. Adv Cancer Res 1979;29:175-274.

3 Kaplowitz N. Physiological significance of glutathione S-transferases. Am J Physiol 1980;239:G439-G444.

4 Mannervik B. The isoenzymes of glutathione S-transferases. Adv Enzymol Rel Areas Mol Biol 1985;57:357-417.

5 Strange RC, Faulder FG, Davies BA, et al. The human glutathione S-transferases: Studies on the tissue distribution and genetic variation of the GST1, GST2 and GST3 isoenzymes. Ann Hum Genet 1984;48:11-20.

6 Strange RC, Davis BA, Faulder CG, Cotton W, Bain AD Hopkinson DA, Hume $R$. The human glutathione S-transferases: Developmental aspects of GST1, GST2 and GST3 loci. Biochem Genet 1985;23:1011-28.

7 Adachi Y, Horii K, Takahashi Y, Tanihata M, Ohba Y, Yamamoto T. Serum glutathione S-transferase activity in liver diseases. Clin Chim Acta 1980;106:243-55.

8 Beckett GJ, Dyson EH, Chapman BJ, Templeton AJ, Hayes JD. Plasma glutathione S-transferases measured by radioimmunoassay: a sensitive index of hepatocellular damage in man. Clin Chim Acta 1985;28:11-9.

9 Howie AF, Patrick AW, Fisher BM, Collier A, Frier BM Beckett GJ. Plasma hepatic glutathione S-transferase concentrations after insulin-induced hypoglycaemia in normal subjects and diabetic patients. Diabetic Med 1989;6:224-7.

10 Beckett CJ, Hayes PC, Hussey AJ, Bouchier IAD, Hayes JD. Plasma glutathione S-transferase measurements in patients with alcoholic cirrhosis. Clin Chim Acto 1987;169:85-90.

11 Hayes PC, Hussey AJ, Keating J, Bouchier IAD, Williams R, Beckett GJ, Hayes JD. Glutathione S-transferase levels in autoimmune chronic active hepatitis: a more sensitive autoimmune chronic active hepatitis: a more sensitive index of hepatocellular damage than as
aminase. Clin Chim Acta 1988;172:211-6.

12 Beckett GJ, Foster GR, Hussey AJ, et al. Plasma Glutathione $S$-transferases and $F$-protein are more sensitive than alanine aminotransferase as markers of paracetamol (acetaminophen)-induced liver damage. Clin Chem 1989;35:2186-9.

13 Hall A, Foster S, Proctor SJ, Cattan AR. Purification and characterisation of a pi class glutathione $\mathrm{S}$-transferase from human leukaemic cells. $\mathrm{Br} \quad \mathrm{f}$ Haematol 1990;76:494-500.

14 Randall BJ, Angus B, Akiba R, et al. Glutathione S-transferase (placental) as a marker of transformation in the human cervix uteri: an immunohistochemical study. $\mathrm{Br} \mathcal{F}$ Cancer 1990;62:614-8.

15 Terrier P, Townsend AJ, Coindre JM, Triche TJ, Cowan KH An immunohistochemical study of $\mathrm{Pi}$ class Glutathione An immunohistochemical study of Pi class Glutathione Pathol 1990;137:845-53.

16 Harrison DJ, May L, Hayes PC, Haque MM, Hayes JD. Glutathione S-transferases in alcoholic liver disease. Gut 1990;31:909-12. 
17 May L, Harrison DJ. Glutathione S-transferase immunolocalisation in human liver: studies on ontogeny, cirrhosis and neoplasia. F Pathol 1991;163:178.

18 Desmet VJ. Cholangiopathies: Past, present, and future. Semin Liver Dis 1987;7:67-76.

19 Ishak KG, Sharp HL. Developmental abnormalities and liver disease in childhood. In: MacSween RNM, Anthony PP, Scheuer PJ, eds. Pathology of the liver. 2nd. ed. Edinburgh: Churchill Livingstone, 1987:66-98. 20 MacSween RNM, Burt AD. Pathology of the intrahepatic
bile ducts. In: Anthony PP, MacSween RNM, eds. Recent advances in pathology Vol 14. 1st ed, Edinburgh,
Churchill Livingstone, 1989:161-83.

21 Raweily EA, Gibson AAM, Burt AD. Abnormalities of intrahepatic bile ducts in extrahepatic biliary atresia. Histopathology 1990;17:521-7.

22 Hiley C, Fryer F, Bell J, Hume R, Strange RC. The human glutathione S-transferases. Biochem $\mathcal{f}$ 1988;254:255-9.

23 Hayes PC, Harrison DJ, Bouchier IAD, McLellan L Hayes JD. Cystolic and microsomal glutathione S-transferase isoenzymes in normal human liver and intestinal epithelium. Gut 1989;30:854-9.

24 Brough JA, Bernstein J. Conjugated hyperbilirubinaemia in early infancy. Hum Pathol 1974;5:507-16. 